\title{
759.
}

\section{ILLUSTRATION OF A THEOREM IN THE THEORY OF EQUATIONS.}

[From the Messenger of Mathematics, vol. xI. (1882), pp. 111-113.]

THE knowledge of the value of an unsymmetrical function of the roots of a numerical equation adds something to what is given by the equation itself; but it may or may not add anything to what is given by the equation itself in regard to each root separately. If, for instance, $\alpha, \beta, \gamma$ being the roots of a cubic equation, it is known that $\alpha^{2} \beta+\beta^{2} \gamma+\gamma^{2} \alpha=a$ given value $k$, then $\alpha, \beta, \gamma$ must denote the roots, taken not in any order whatever, nor yet in a uniquely determinate order, but with a certain restriction as to order, viz. if the roots in a certain order are $a, b, c$, these roots being such that $a^{2} b+b^{2} c+c^{2} a=k$, then clearly the relation in question $\alpha^{2} \beta+\beta^{2} \gamma+\gamma^{2} \alpha=k$, will be satisfied if $\alpha, \beta, \gamma=a, b, c$, or $=b, c, a$, or $=c, a, b$ (but not if $\alpha, \beta, \gamma=b, a, c$, or = either of the remaining two arrangements); the relation thus allows $\alpha$ to be $=a$, or $=b$, or $=c$; that is, $\alpha$ is =any one at pleasure of the roots of the cubic equation, and it is thus determined by the cubic equation, and not by any inferior equation; but $\alpha$ being known, the other two roots $\beta$ and $\gamma$ will be uniquely, and therefore rationally, determined.

It is worth while to see how the result works out; suppose, for greater simplicity, the cubic equation is $x^{3}-7 x+6=0$ having roots $(1,2,-3)$, and that the given relation is $\alpha^{2} \beta+\beta^{2} \gamma+\gamma^{2} \alpha=-1$, then the cubic equation gives

$$
\alpha+\beta+\gamma=0, \quad \alpha \beta+\alpha \gamma+\beta \gamma=-7, \quad \alpha \beta \gamma=-6,
$$

and we have, besides, the relation in question

eliminating $\gamma$ we have

$$
\alpha^{2} \beta+\beta^{2} \gamma+\gamma^{2} \alpha=-1
$$

$$
\alpha^{2}+\alpha \beta+\beta^{2}=7, \quad \alpha \beta(\alpha+\beta)=6, \quad \alpha^{3}+3 \alpha^{2} \beta-\beta^{3}+1=0 ;
$$

or, as it is convenient to write these equations,

$$
\begin{aligned}
& \beta^{2}+\alpha \beta+\alpha^{2}-7=0, \\
& \beta^{2}+\alpha \beta-\frac{6}{\alpha}=0, \\
& \beta^{3}-3 \alpha^{2} \beta-\alpha^{3}-1=0 .
\end{aligned}
$$


If from these equations we eliminate $\beta$, we obtain two equations in $\alpha$, which it might be supposed would determine $\alpha$ uniquely; but, by what precedes, $\alpha$ is any root at pleasure of the cubic equation and can thus be determined only by the cubic equation itself, and it follows that any equation obtained by the elimination of $\beta$ must contain as a factor the cubic function $\alpha^{3}-7 \alpha+6$, and be thus of the form $M\left(\alpha^{3}-7 \alpha+6\right)=0$, where $M$ is a function of $\alpha$; one result of the elimination is $\alpha^{3}-7 \alpha+6=0$, and every other result is of the form just referred to, $M\left(\alpha^{3}-7 \alpha+6\right)=0$; hence we have definitely $\alpha^{3}-7 \alpha+6=0$, viz. the roots of the equation $M=0$ do not apply to the question.

In verification, observe that the first and second equations give $\alpha^{2}-7=\frac{6}{\alpha}$, that is, $\alpha^{2}-6 \alpha+7=0$. To eliminate $\beta$ from the first and third equations we first find

or say

$$
\alpha \beta^{2}+\left(4 \alpha^{2}-7\right) \beta+\alpha^{3}+1=0,
$$

$$
\beta^{2}+\left(4 \alpha-\frac{7}{\alpha}\right) \beta+\alpha^{2}+\frac{1}{\alpha}=0
$$

and combining herewith the first equation

we obtain

$$
\beta^{2}+\alpha \beta+\alpha^{2}-7=0,
$$

$$
\beta\left(3 \alpha-\frac{7}{\alpha}\right)+7+\frac{1}{\alpha}=0,
$$

that is,

$$
\beta=\frac{7 \alpha+1}{-3 \alpha^{2}+7}
$$

substituting in the first equation,

that is,

$$
\begin{aligned}
& (7 \alpha+1)^{3} . \\
+ & \alpha(7 \alpha+1)\left(-3 \alpha^{2}+7\right) \\
+\quad & \left(\alpha^{2}-7\right)\left(-3 \alpha^{2}+7\right)^{2}=0,
\end{aligned}
$$

or, dividing by 3 ,

$$
\begin{aligned}
& \begin{array}{lll}
49 & 14 & 1
\end{array} \\
& -21-3+49+7 \\
& 90-105 \quad+343 \quad-343 \\
& 90-126-3+441+21-342 \text {, }
\end{aligned}
$$

which, in fact, is

$$
3 \alpha^{6}-42 \alpha^{4}-\alpha^{3}+147 \alpha^{2}+7 \alpha-114=0,
$$

$$
\left(\alpha^{3}-7 \alpha+6\right)\left(3 \alpha^{3}-21 \alpha-19\right)=0,
$$

of the form in question $M\left(\alpha^{3}-7 \alpha+6\right)=0$. Thus $\alpha$ has any one at pleasure of the three values $1,2,-3$, but $\alpha$ being known we have $\beta=\frac{7 \alpha+1}{-3 \alpha^{2}+7}$, and thence

$$
\gamma=-\alpha+\frac{-7 \alpha-1}{-3 \alpha^{2}+7},=\frac{3 \alpha^{3}-14 \alpha-1}{-3 \alpha^{2}+7}
$$

in particular, as $\alpha=1$, then $\beta=2$ and $\gamma=-3$. 